Article

\title{
Existence and local stability of stationary solutions for nonlinear Gilpin Ayala competition model with Dirichlet boundary value
}

\author{
Ruofeng Rao ${ }^{1,2}$ \\ 1 Department of Mathematics, Chengdu Normal University, Chengdu 61130, China; ruofengrao@163.com or \\ ruofengrao@cdnu.edu.cn \\ 2 Institute of Financial Mathematics, Chengdu Normal University, Chengdu 61130, China
}

\begin{abstract}
In this paper, the existence of two nontrivial stationary solutions for the nonlinear Gilpin Ayala two species competition model is given by using the mountain pass lemma, and the local stability criterion of the trivial solution is given by using Lyapunov function method. Based on the local stability criterion, we give some suggestions on how to avoid the population extinction. This is, when the population is on the verge of extinction, we should try our best to avoid the diffusion behavior and reduce the diffusion coefficient, otherwise the species are easy to go extinct. Numerical example shows the effectiveness of the proposed method.
\end{abstract}

Keywords: Gilpin Ayala competition model; Lyapunov function; Mountain Pass Lemma; Palais Smale condition; Dirichlet boundary value

\section{Introduction}

In 1920, Lotka and Volterra proposed the famous population competition model ([1,2]):

$$
\left\{\begin{array}{l}
\dot{x}_{1}(t)=x_{1}(t)\left[b_{1}-a_{11} x_{1}(t)-a_{12} x_{2}(t)\right], \\
\dot{x}_{2}(t)=x_{2}(t)\left[b_{2}-a_{21} x_{1}(t)-a_{22} x_{2}(t)\right],
\end{array}\right.
$$

where $x_{i}(t)$ represents the population density of the $i$ th population at time $t(i=1,2), b_{i}>0$ represents the birth rate of the population of the $i$ th population, $a_{i j}>0$ represents the competition parameter of two populations, which is recognized and cited by many scholars. Diffusion is usually considered reasonably, for example in the reference [3] and related references:

$$
\left\{\begin{array}{rlrl}
\frac{\partial u_{1}}{\partial t} & =d_{1} \Delta u_{1}+u_{1}(t)\left[b_{1}-a_{11} u_{1}(t)-a_{12} u_{2}(t)\right], & & x \in \Omega, t>0 \\
\frac{\partial u_{2}}{\partial t} & =d_{2} \Delta u_{2}+u_{2}(t)\left[b_{2}-a_{21} u_{1}(t)-a_{22} u_{2}(t)\right], & & x \in \Omega, t>0 \\
\frac{\partial u_{1}}{\partial v} & =\frac{\partial u_{2}}{\partial v}=0, \quad x \in \partial \Omega, t>0, & \\
u_{1}(x, 0) & =u_{0}(x), u_{2}(x, 0)=v_{0}(x), \quad x \in \Omega . &
\end{array}\right.
$$

and 


$$
\left\{\begin{array}{rlrl}
\frac{\partial u}{\partial t} & =\Delta\left[\left(d_{1}+a_{11} u+a_{12} v\right) u\right]+\mu_{1} u\left(1-u-a_{1} v\right), & & x \in \Omega, t>0 \\
\frac{\partial v}{\partial t} & =\Delta\left[\left(d_{2}+a_{21} u+a_{22} v\right) v\right]+\mu_{2} v\left(1-v-a_{2} u\right), \quad & & x \in \Omega, t>0 \\
\frac{\partial u}{\partial v} & =\frac{\partial v}{\partial v}=0, \quad x \in \partial \Omega, t>0, & \\
u(x, 0) & =u_{0}(x), v(x, 0)=v_{0}(x), \quad x \in \Omega .
\end{array}\right.
$$

In 2017, Yuanyuan Liu and Youshan Tao studied the linear competition model of cross diffusion of two populations under Neumann boundary conditions ([4]):

$$
\left\{\begin{aligned}
\frac{\partial u}{\partial t} & =\Delta\left[\left(d_{1}+a_{12} v\right) u\right]+\mu_{1} u\left(1-u-a_{1} v\right), \quad x \in \Omega, t>0 \\
0 & =\Delta v+\mu_{2} v\left(1-v-a_{2} u\right), \quad x \in \Omega, t>0 \\
\frac{\partial u}{\partial v} & =\frac{\partial v}{\partial v}=0, \quad x \in \partial \Omega, t>0 \\
u(x, 0) & =u_{0}(x), \quad x \in \Omega .
\end{aligned}\right.
$$

In 1973, Gilpin and Ayala found that the linear competition model was not consistent with the experimental results ([5]). Through accurate data analysis, they proposed a nonlinear competition model of two populations:

$$
\left\{\begin{array}{l}
\dot{x}_{1}(t)=x_{1}(t)\left[b_{1}-a_{11} x_{1}^{\theta_{1}}(t)-a_{12} x_{2}(t)\right], \\
\dot{x}_{2}(t)=x_{2}(t)\left[b_{2}-a_{21} x_{1}(t)-a_{22} x_{2}^{\theta_{2}}(t)\right],
\end{array}\right.
$$

where $\theta_{1}, \theta_{2}$ represents the nonlinear density constraint parameter. As pointed out in [6-9], when the parameter $\theta_{i}$ is much less than 1 , the nonlinear density constrained model can well simulate the population ecology of Drosophila melanogaster, and the diffusion type Gilpin Ayala competition model under Neumann boundary value condition has also been studied by scholars:

$$
\left\{\begin{array}{l}
\frac{\partial u_{1}}{\partial t}=d_{1} \Delta u_{1}+u_{1}\left(b_{1}-a_{11} u_{1}^{\theta_{1}}-a_{12} u_{2}\right) \\
\frac{\partial u_{2}}{\partial t}=d_{2} \Delta u_{2}+u_{2}\left(b_{2}-a_{22} u^{\theta_{2}}-a_{21} u_{1}\right) \\
\frac{\partial u_{1}}{\partial v}=\frac{\partial u_{2}}{\partial v}=0, \quad x \in \partial \Omega, t>0
\end{array}\right.
$$

where $\mu_{1}, \mu_{2}, a, b, c$ and $d$ all are positive numbers.

It is noted that the diffusion ecosystem with Neumann boundary value has been widely studied $([3,4,8]$ and related references), but the diffusive ecosystem under Dirichlet boundary value is rarely studied. In fact, the Drichlet boundary value diffusion ecosystem can better reflect the actual population ecology. Therefore, this paper will study the dynamic behavior of nonlinear Gilpin Ayala competition model with Dirichlet zero boundary value, I will give the existence of two nonzero steady-state solutions for this model. Recently, the author has studied the double positive solutions of the following delay feedback Gilpin-Ayala competition model in [20], where the global stability of the positive solution was presented in [20].

$$
\left\{\begin{array}{l}
\frac{\partial u_{1}}{\partial t}=d_{1} \Delta u_{1}+u_{1}\left(b_{1}-a_{11} u_{1}^{\theta_{1}}-a_{12} u_{2}\right)+k_{1}(r(t))\left[u_{1}-u_{1}\left(t-\tau_{1}(t), x\right)\right]+\chi_{1}, \quad t \geqslant 0, x \in \Omega \\
\frac{\partial u_{2}}{\partial t}=d_{2} \Delta u_{2}+u_{2}\left(b_{2}-a_{21} u_{1}-a_{22} u_{2}^{\theta_{2}}\right)+k_{2}(r(t))\left[u_{2}-u_{2}\left(t-\tau_{2}(t), x\right)\right]+\chi_{2}, \quad t \geqslant 0, x \in \Omega \\
u_{1}(t, x)=u_{2}(t, x)=0, \quad t \geqslant 0, x \in \partial \Omega
\end{array}\right.
$$

So, in this paper, I only study the stability of zero solution, avoiding duplication with another article of mine 
This paper, I denote by $\lambda_{1}$ the first positive eigenvalue of the Laplace operator $-\Delta$ in $H_{0}^{1}(\Omega)$. Denote by $\|u\|=\sqrt{\int_{\Omega}|\nabla u(x)|^{2} d x}$ the norm of Sobolev space $H_{0}^{1}(\Omega)$.

\section{Preparation}

Consider the nonlinear Gilpin-Ayala competition model under Dirichlet boundary value:

$$
\left\{\begin{array}{l}
\frac{\partial u_{1}}{\partial t}=d_{1} \Delta u_{1}+u_{1}\left(b_{1}-a_{11} u_{1}^{\theta_{1}}-a_{12} u_{2}\right), \quad t \geqslant 0, x \in \Omega, \\
\frac{\partial u_{2}}{\partial t}=d_{2} \Delta u_{2}+u_{2}\left(b_{2}-a_{21} u_{1}-a_{22} u_{2}^{\theta_{2}}\right), \quad t \geqslant 0, x \in \Omega, \\
u_{1}(t, x)=u_{2}(t, x)=0, \quad t \geqslant 0, x \in \partial \Omega \\
u_{1}(0, x)=\xi_{1}(x), \quad u_{2}(0, x)=\xi_{2}(x),
\end{array}\right.
$$

where $\Omega$ is a domain in $\mathbb{R}^{3}$ with the smooth boundary $\partial \Omega$.

Remark 1. Here, we assume $\Omega \subset \mathbb{R}^{3}$. And if two species live in two dimensional plane, we can assume $u_{i}(t, x)=u_{i}\left(t, x_{1}, x_{2}, x_{3}\right)=u_{i}\left(t, x_{1}, x_{2}, \cdot\right)$, independent of the third dimension, where $x=\left(x_{1}, x_{2}, x_{3}\right)^{T} \in \Omega$.

Besides, I need Mountain Pass Lemma as follows ([12]).

Lemma 2.1 (Mountain Pass Lemma without the (PS) condition). Let $X$ is a Banach space, $\Psi \in C^{1}(X, \mathbb{R})$, satisfying $\Psi(0)=0$, and there exists $\rho>0$ such that $\left.\Psi\right|_{\partial B_{\rho}(0)} \geqslant \alpha>0$. Besides, there is $e \in X \backslash \overline{B_{\rho}(0)}$ such that $\Psi(e) \leqslant 0$. Let $\Gamma$ be the set of all paths connecting 0 and $e$. That is,

$$
\Gamma=\left\{\psi \in C\left([0,1], H_{0}^{1}(\Omega)\right): \psi(0)=0, \psi(1)=e\right\} .
$$

Set

$$
c_{*}=\inf _{\psi \in \Gamma} \max _{s \in[0,1]} \Psi(\psi(s)) .
$$

Then $c_{*} \geqslant \alpha$, and $\Psi$ possesses a critical sequence on $c_{*}$.

Remark 2. Lemma 2.1 is the Mountain Pass Lemma without the (PS) condition (see,e.g. [11,12]). If, in addition, $\Psi$ satisfies the (PS) condition, then $c_{*}$ is a critical value of $\Psi$.

\section{Main results}

As pointed out in [6-9], As pointed out in [6-9], when the parameter $\theta_{i}$ is much less than 1, the nonlinear density constrained model can well simulate the population ecology of Drosophila melanogaster. So I assume $\theta_{i} \in(0,1)$, and

(H1) For each $i \in\{1,2\}$, there are positive numbers $p_{i}, q_{i}$ such that $\frac{p_{i}}{q_{i}}-2=\theta_{i} \in(0,1)$, where $p_{i}$ and $q_{i}$ are a pair of Coprime odd numbers.

Theorem 3.1. Suppose (H1) holds, $b_{i}<d_{i} \lambda_{1}$ and $0<\theta_{i}<1, \forall i=1$, 2. Then the system (2.1) possesses at least three stationary solutions $(0,0),\left(u_{1 *}(x), 0\right)$ and $\left(0, u_{2 *}(x)\right)$, where $u_{i *}(x) \neq 0, \forall i=1,2$.

Proof. Firstly, $(0,0)$ is a trivial solution of the system (2.1).

Next, if $\left(u_{1}(x), 0\right)$ is a stationary solution of the system (2.1),

$$
\left\{\begin{array}{l}
d_{1} \Delta u_{1}(x)+b_{1} u_{1}(x)-a_{11} u_{1}(x)^{1+\theta_{1}}=0, \quad \text { a.e. } x \in \Omega, \\
u_{1}(x)=0, \quad x \in \partial \Omega
\end{array}\right.
$$

Similarly, if $\left(0, u_{2}(x)\right)$ is a stationary solution of the system $(2.1)$,

$$
\left\{\begin{array}{l}
d_{2} \Delta u_{2}(x)+b_{2} u_{2}(x)-a_{22} u_{2}(x)^{1+\theta_{2}}=0 \\
u_{2}(x)=0, \quad x \in \partial \Omega
\end{array}\right.
$$

Obviously,

$$
J\left(u_{1}\right)=\frac{1}{2} d_{1}\left\|u_{1}\right\|^{2}-\frac{1}{2} b_{1} \int_{\Omega} u_{1}^{2} d x+\frac{a_{11}}{2+\theta_{1}} \int_{\Omega} u_{1}^{2+\theta_{1}} d x
$$


is the functional corresponding to the equation (3.1), and $J \in C^{1}\left(H_{0}^{1}(\Omega), \mathbb{R}^{1}\right)$.

Besides, $J(0)=0$. And Sobolev embedding theorem yields that there is $c>0$ such that

$$
\begin{aligned}
J\left(u_{1}\right) & =\frac{1}{2} d_{1}\left\|u_{1}\right\|^{2}-\frac{1}{2} b_{1} \int_{\Omega} u_{1}^{2} d x+\frac{a_{11}}{2+\theta_{1}} \int_{\Omega} u_{1}^{2+\theta_{1}} d x \geqslant \frac{1}{2} d_{1}\left\|u_{1}\right\|^{2}-\frac{b_{1}}{2 \lambda_{1}}\left\|u_{1}\right\|^{2}-\frac{a_{11}}{2+\theta_{1}} \int_{\Omega}\left|u_{1}\right|^{2+\theta_{1}} d x \\
& \geqslant \frac{1}{2} d_{1}\left(1-\frac{b_{1}}{d_{1} \lambda_{1}}\right)\left\|u_{1}\right\|^{2}-\frac{c a_{11}}{2+\theta_{1}}\left\|u_{1}\right\|^{2+\theta_{1}} .
\end{aligned}
$$

Let $\rho>0$ small enough such that

$$
\left.J\right|_{\partial B_{\rho}(0)} \geqslant \alpha,
$$

where $\alpha=\frac{1}{2} d_{1}\left(1-\frac{b_{1}}{d_{1} \lambda_{1}}\right) \rho^{2}-\frac{c a_{11}}{2+\theta_{1}} \rho^{2+\theta_{1}}>0$. Denote by $\varphi_{1}(x)>0$ the eigenfunction of $\lambda_{1}$, satisfying $\left\|\varphi_{1}\right\|=1$ $([11,17])$. Then

$$
J\left(-s \varphi_{1}\right)=\frac{1}{2} d_{1}\left\|-s \varphi_{1}\right\|^{2}-\frac{1}{2} b_{1} \int_{\Omega}\left(-s \varphi_{1}\right)^{2} d x+\frac{a_{11}}{2+\theta_{1}} \int_{\Omega}\left(-s \varphi_{1}\right)^{2+\theta_{1}} d x \rightarrow-\infty, \quad s \rightarrow+\infty,
$$

Thereby, there is a $s_{0}$ such that $s_{0}>\rho$ and $J\left(-s_{0} \varphi_{1}\right)<0$, where $\left\|-s_{0} \varphi_{1}\right\|=s_{0}>\rho$.

Let $\Gamma$ be the set of all paths connecting 0 and $-s_{0} \varphi_{1}$, i.e.,

$$
\Gamma=\left\{\psi \in C\left([0,1], H_{0}^{1}(\Omega)\right): \psi(0)=0, \psi(1)=-s_{0} \varphi_{1}\right\} .
$$

Set

$$
c_{0}=\inf _{\psi \in \Gamma} \max _{s \in[0,1]} J(\psi(s)) .
$$

then

$$
c_{0} \geqslant \frac{1}{2} d_{1}\left(1-\frac{b_{1}}{d_{1} \lambda_{1}}\right) \rho^{2}-\frac{c a_{11}}{2+\theta_{1}} \rho^{2+\theta_{1}}>0,
$$

Lemma 2.1 yields that there is a sequence $\left\{u_{1 n}\right\}_{n=1}^{\infty} \subset H_{0}^{1}(\Omega)$ such that

$$
J\left(u_{1 n}\right) \rightarrow c_{0}, \quad \text { and } \quad J^{\prime}\left(u_{1 n}\right) \rightarrow 0, \quad n \rightarrow \infty .
$$

Below, similarly as those of [18], I will prove the sequence $\left\{u_{1 n}\right\}_{n=1}^{\infty} \subset H_{0}^{1}(\Omega)$ satisfying (3.10) must be bounded.

In fact, (3.10) yields

$$
\frac{1}{2} d_{1}\left\|u_{1 n}\right\|^{2}-\frac{1}{2} b_{1} \int_{\Omega} u_{1 n}^{2} d x+\frac{a_{11}}{2+\theta_{1}} \int_{\Omega} u_{1 n}^{2+\theta_{1}} d x=c_{0}+o(1)
$$

and

$$
d_{1}\left\|u_{1 n}\right\|^{2}-b_{1} \int_{\Omega} u_{1 n}^{2} d x+a_{11} \int_{\Omega} u_{1 n}^{2+\theta_{1}} d x=\left\langle J^{\prime}\left(u_{1 n}\right), u_{1 n}\right\rangle
$$

and for $\varepsilon>0$ small enough suc that there exits a $n$ big enough such that

$$
\left|\left\langle J^{\prime}\left(u_{1 n}\right), u_{1 n}\right\rangle\right| \leqslant \varepsilon\left\|u_{1 n}\right\|
$$

So I have

$$
d_{1}\left(\frac{1}{2}-\frac{1}{2+\theta_{1}}\right)\left(1-\frac{b_{1}}{d_{1} \lambda_{1}}\right)\left\|u_{1 n}\right\|^{2} \leqslant c_{0}+o(1)-\frac{\varepsilon}{2+\theta_{1}}\left\|u_{1 n}\right\|,
$$

which means the boundedness of $\left\{u_{1 n}\right\}_{n=1}^{\infty}$.

Now I shall prove that the bounded sequence $\left\{u_{1 n}\right\}_{n=1}^{\infty}$ must be compact sequentially. This is only a conventional proof. However, in view of the completeness of the proof, I am willing to give the proof:

In fact, (H1) means $\frac{1}{d_{1}}\left(b_{1} u_{1}(x)-a_{11} u_{1}(x)^{1+\theta_{1}}\right)$ satisfies the Caratheodory condition:

$$
\left|\frac{1}{d_{1}}\left(b_{1} u_{1}(x)-a_{11} u_{1}(x)^{1+\theta_{1}}\right)\right| \leqslant c_{1}+c_{2}\left|u_{1}\right|^{2}, \quad \forall\left(x, u_{1}\right) \in \Omega \times \mathbb{R},
$$


where $c_{1}, c_{2}$ are positive numbers big enough. Due to $\Omega \subset \mathbb{R}^{3}$, the critical Sobolev exponent is 6 , and hence the operator $J^{\prime}: H_{0}^{1}(\Omega) \rightarrow\left(H_{0}^{1}(\Omega)\right)^{*}$ is compact, where the functional

$$
\tilde{J}=\int_{\Omega}\left(\frac{1}{2} b_{1} u_{1}^{2}-\frac{a_{11}}{2+\theta_{1}} u_{1}^{2+\theta_{1}}\right) d x .
$$

Moreover,

$$
\left\langle\tilde{J}^{\prime}\left(u_{1}\right), \varphi\right\rangle=\int_{\Omega}\left(b_{1} u_{1}(x) \varphi-a_{11} u_{1}(x)^{1+\theta_{1}} \varphi\right) d x, \quad \forall \varphi \in H_{0}^{1}(\Omega) .
$$

and then the bounded sequence $\left\{u_{1 n}\right\}_{n=1}^{\infty}$ possesses a subsequence, say, $\left\{u_{1 n}\right\}_{n=1}^{\infty}$, satisfying $J^{\prime}\left(u_{1 n}\right) \rightarrow J^{\prime}\left(u_{1 *}\right)$ in $\left(H_{0}^{1}(\Omega)\right)^{*}, n \rightarrow \infty$, where $u_{1 *} \in H_{0}^{1}(\Omega)$. For any $\varphi \in H_{0}^{1}(\Omega)$,

$$
\left\langle J^{\prime}\left(u_{1 n}\right)-J^{\prime}\left(u_{1 m}\right), \varphi\right\rangle=d_{1} \int_{\Omega}\left(\nabla u_{1 n}-\nabla u_{1 m}\right) \cdot \nabla \varphi d x-\left\langle\tilde{J}^{\prime}\left(u_{1 n}\right)-\tilde{J}^{\prime}\left(u_{1 m}\right), \varphi\right\rangle,
$$

which together with $\left\{u_{1 n}\right\}_{n=1}^{\infty} \subset H_{0}^{1}(\Omega),(3.10)$ and the arbitrariness of $\varphi$ implies

$$
\begin{gathered}
\quad\left\|u_{1 n}-u_{1 m}\right\|^{2} \leqslant\left(\left\|J^{\prime}\left(u_{1 n}\right)\right\|+\left\|J^{\prime}\left(u_{1 m} \|\right)\right\| u_{1 n}-u_{1 m}\|+\| \tilde{J}^{\prime}\left(u_{1 n}\right)-\tilde{J}^{\prime}\left(u_{1 m}\right)\|\| u_{1 n}-u_{1 m} \|\right. \\
\leqslant\left(\left\|J^{\prime}\left(u_{1 n}\right)\right\|+\| J^{\prime}\left(u_{1 m}\|+\| \tilde{J}^{\prime}\left(u_{1 n}\right)-\tilde{J}^{\prime}\left(u_{1 m}\right) \|\right)\left(\left\|u_{1 n}\right\|+\left\|u_{1 m}\right\|\right) \rightarrow 0, \quad n \rightarrow \infty, m \rightarrow \infty,\right.
\end{gathered}
$$

This shows that $\left\{u_{1 n}\right\}_{n=1}^{\infty}$ is compact sequentially. And then there exists a subsequence of $\left\{u_{1 n}\right\}_{n=1}^{\infty}$ convergent to a point in $H_{0}^{1}(\Omega)$, say, $u_{1 *} \in H_{0}^{1}(\Omega)$, Due to $J\left(u_{1 *}\right)=c_{0} \geqslant \frac{1}{2} d_{1}\left(1-\frac{b_{1}}{d_{1} \lambda_{1}}\right) \rho^{2}-\frac{c a_{11}}{2+\theta_{1}} \rho^{2+\theta_{1}}>0$, I see $u_{1 *} \neq 0$, which shows that $\left(u_{1 *}, 0\right) \neq(0,0)$. Similarly, I can similarly prove there is at least another stationary solution $\left(0, u_{2 *}\right) \neq(0,0)$ for the system $(2.1)$.

Theorem 3.2. Under the assumptions of Theorem 3.1 , the zero solution $(0,0)$ is locally asymptotically stable .

Proof. Firstly, the condition $b_{i}<\lambda_{1} d_{i}$ yields,

$$
B<\lambda_{1} D
$$

where

$$
D=\left(\begin{array}{cc}
d_{1} & 0 \\
0 & d_{2}
\end{array}\right), B=\left(\begin{array}{cc}
b_{1} & 0 \\
0 & b_{2}
\end{array}\right) .
$$

Next, consider the following linear system:

Consider the Lyapunov function:

$$
\left\{\begin{array}{c}
\frac{\partial u_{1}}{\partial t}=d_{1} \Delta u_{1}+b_{1} u_{1}, \quad t \geqslant 0, x \in \Omega \\
\frac{\partial u_{2}}{\partial t}=d_{2} \Delta u_{2}+b_{2} u_{2}, \quad t \geqslant 0, x \in \Omega \\
u_{1}(t, x)=u_{2}(t, x)=0, \quad t \geqslant 0, x \in \partial \Omega \\
u_{1}(0, x)=\xi_{1}(x), \quad u_{2}(0, x)=\xi_{2}(x)
\end{array}\right.
$$

The condition (3.14) yields

$$
V=\int_{\Omega}\left(u_{1}^{2}+u_{2}^{2}\right) d x .
$$

$$
\begin{aligned}
\left.\frac{d V}{d t}\right|_{(3.16)} & =\int_{\Omega}\left(2 d_{1} u_{1} \Delta u_{1}+2 b_{1} u_{1}^{2}+2 d_{2} u_{2} \Delta u_{2}+2 b_{2} u_{2}^{2}\right) d x \\
& \leqslant \int_{\Omega} u^{T}\left(-2 \lambda_{1} D+2 B\right) u d x \leqslant 0
\end{aligned}
$$

where $u=\left(u_{1}, u_{2}\right)^{T}$. Then (3.17) yields that the zero solution $(0,0)$ of the linear system (3.16) is asymptotically stable ([19]). And hence, the zero solution $(0,0)$ of the nonlinear system (2.1) is locally asymptotically stable. 


\section{Numerical example}

Example 4.1. In the system (2.1), I assume $\Omega=\left(-\frac{1}{2}, \frac{1}{2}\right) \times\left(-\frac{1}{2}, \frac{1}{2}\right) \times\left(-\frac{1}{2}, \frac{1}{2}\right)$, then $\lambda_{1} \geqslant 3$ ([11, Remark 14]). Set $\theta_{1}=\frac{1}{3}, \theta_{2}=\frac{1}{5}$, then the condition (H1) is satisfied. Assume, in addition,

$$
D=\left(\begin{array}{cc}
0.6 & 0 \\
0 & 0.5
\end{array}\right), B=\left(\begin{array}{cc}
1.5 & 0 \\
0 & 1.2
\end{array}\right),
$$

then $b_{i}<d_{i} \lambda_{1}$ and $0<\theta_{i}<1, \forall i=1,2$. Theorem 3.1 tells that the system (2.1) possesses at least three stationary solutions $(0,0),\left(u_{1 *}(x), 0\right)$ and $\left(0, u_{2 *}(x)\right)$, where $u_{i *}(x) \neq 0, \forall i=1,2$. Moreover, Theorem 3.2 yields that the zero solution $(0,0)$ of the system $(2.1)$ is locally asymptotically stable.

\section{Conlusions}

In this paper, the existence of two nontrivial stationary solutions of the nonlinear Gilpin-Ayala model of two species competition is derived by using the mountain pass lemma. The local stability criteria of the trivial solutions are given by using the Lyapunov function method. The local stability conclusion of the double zero solution fully indicates that when the population is on the verge of extinction, the diffusion behavior should be avoided and the diffusion coefficient should be reduced, otherwise the species will be prone to extinction

Funding: The work is supported by the Application basic research project of science and Technology Department of Sichuan Province (No. 2020YJ0434) and the Major scientific research projects of Chengdu Normal University in 2019 (No. CS19ZDZ01).

Conflicts of Interest: The author declares no conflict of interest.

\section{References}

1. Lotka A. Elements of physical biology. Williams and Wilkins, Baltimore, Md., 1924.

2. Volterra Vito. Lecons sur la Theorie Mathematique de la Lutte pour La Vie. Gauthier-Villars, Paris, 1931.

3. Nanako Shigesada, Kohkichi Kawasaki, Ei Teramoto. Spatial segregation of interacting species. Journal of Theoretical Biology, Volume 79, Issue 17 July 1979Pages 83-99

4. Yuanyuan Liu, Youshan Tao. Dynamics in a parabolic-elliptic two-species population competition model with cross-diffusion for one species. Journal of Mathematical Analysis and Applications, 2017, 456(11), 1-15.

5. Gilpin, M.E., Ayala, F.J., Global models of growth and competition. Proceedings of the National Academy of Sciences of the United States of America, 70 (1973) 3590-3593

6. Michael E. Gilpin, Francisco J. Ayala. Schoener's model and Drosophila competition. Theoretical Population Biology,1976 , 9(1), 12-14

7. William R. Thomas,Mark J. Pomerantz,Michael E. Gilpin. Chaos, Asymmetric Growth and Group Selection for Dynamical Stability. Ecology, 1980, 61,1312-1320. DOI: 10.2307/1939039.

8. Meixiang Chen, Xizhuang Xie. Bi-stability of two-species competition model with reaction diffusion. Jounral of Huaqiao University (Natural Science), 2020, 41(2), 268-271.

9. Moore, Christopher M.,Catella, Samantha A.,Abbott, Karen C. Population dynamics of mutualism and intraspecific density dependence: How $\theta$-logistic density dependence affects mutualistic positive feedback. Ecological Modelling Volume 368, 24 January 2018, Pages 191-197

10. Ruofeng Rao. Positive Solution for the Dirichlet Zero-Boundary Value Problem (In Chinese). College Mathematics,2010,26(02):146-152.

11. Ruofeng Rao. Stability Analysis of Nontrivial Stationary Solution and Constant Equilibrium Point of Reaction-Diffusion Neural Networks with Time Delays under Dirichlet Zero Boundary Value. Preprints 2020, 2020040277 (doi: 10.20944/preprints202004.0277.v5).

12. Haim Brezis, Louis Nirenberg. Remarks on finding critical points. Communications on Pure \& Applied Mathematics, 44 (1991), 939-963.

13. Willem M. Minimax theorems. Berlin: Birkhauser, 1996.

14. Ji Y, Chizeck HJ. Controllability, stabilizability, and continuous-time Markovian jump linear quadratic control. IEEE Trans Autom Control 1990;35:777-88.

15. Ruofeng Rao, Shouming Zhong, Xiongrui Wang. Stochastic stability criteria with LMI conditions for Markovian jumping impulsive BAM neural networks with mode-dependent time-varying delays and 
nonlinear reaction-diffusion. Communications in Nonlinear Science and Numerical Simulation, Volume 19, Issue 1January 2014Pages 258-273

16. Ruofeng Rao and Shouming Zhong. Impulsive control on delayed feedback chaotic financial system with Markovian jumping. Advances in Difference Equations, 2020 2020:50

17. Ruofeng Rao, Xiongrui Wang.Infinitely Many Solutions for the Resonant Quasi-linear Equation Without Landesman-Lazer Conditions(In Chinese). Acta Mathematica Scientia, Ser. A, 2012,32(04):744-752.

18. Ruofeng Rao.On the Elliptic Equations With the First Eigenvalue,Involving the Critial Sobolev Exponents (In Chinese). Advances In Mathematics (China), 2004(06):703-711.

19. Liao Xiaoxin. Theory, method and application of stability. Huazhong Science and Technology Press, China, 2004.

20. Ruofeng Rao. Unique Existence of Globally Asymptotical Input-to-State Stability of Positive Stationary Solution for Impulsive Gilpin-Ayala Competition Model with Diffusion and Delayed Feedback under Dirichlet Zero Boundary Value. Preprints 2020, 2020090052 (doi: 10.20944/preprints202009.0052.v1). 